\title{
Prognostic value of frontal functional neuroimaging in late-onset severe major depression
}

\author{
VÍCTOR NAVARRO, CRISTÓBAL GASTÓ, FRANCISCO LOMEÑA, \\ XAVIER TORRES, JOSÉ J. MATEOS, MARÍA J. PORTELLA, \\ GUILLEM MASANA and TEODORO MARCOS
}

\author{
Background There is growing \\ evidence of a relationship between frontal \\ neuroimaging and neuropsychological \\ abnormalities and the physiopathology \\ and course of late-onset major \\ depression.
}

\begin{abstract}
Aims To assess acute antidepressant response in late-onset major depression in relation to baseline frontal perfusion ratios.
\end{abstract}

\section{Method A ${ }^{99 m T c H M P A O ~ s i n g l e ~}$ photon emission computed tomographic brain scan was performed in medication- free patients with late-onset major depression, who were then included in a \\ 12-week antidepressant treatment regimen. Logistic regression was used to define a predictive model of non- remission.}

\section{Results A total of 47 patients completed the study, 34 of whom were classed as remitters and 13 as non- remitters. The variable left anterior fronto- cerebellar perfusion ratio had a global predictive power of $87 \%$. Analysing this variable together with the baseline variables age of onset and duration of index episode, the predictive power of the model rose to $94 \%$.}

Conclusions Our study suggests that a specific frontal functioning could predict the acute antidepressant response in lateonset severe major depression.

\section{Declaration of interest None.}

Neuropsychological studies (Alexopoulos et al, 2000), structural neuroimaging studies (Alexopoulos et al, 2002) and functional neuroimaging studies (Kumar et al, 2002; Navarro et al, 2002) have suggested the possible involvement of the frontal lobes in the physiopathology of late-onset major depression. Various structural neuroimaging studies have stressed the possible influence of these frontal alterations on the course of the disorder (Simpson et al, 1998; Alexopoulos et al, 2002). To our knowledge, no prognostic study as yet has used functional neuroimaging. Following on from the vascular depression concept defined by Alexopoulos et al (1997), the hypothesis we put forward here is that the greater the frontal hypoperfusion, the poorer the clinical evolution of late-onset major depression. After establishing in an earlier study (Navarro et al, 2002) that perfusion abnormalities detected during active depression in elderly patients in the left anterior frontal region disappeared in remission, the main aim of our study was to assess in a new sample the acute response to antidepressant medication of patients with late-onset major depression in relation to baseline frontal perfusion ratios.

\section{METHOD}

\section{Sample}

The study was conducted at the Hospital Clínic of Barcelona. Participants were recruited from in-patients and out-patients with a diagnosis of major depressive disorder, aged 60 years or over; all were right-handed. Only people with late-onset depression were included, i.e. those in whom depression had begun after the age of 50. To be eligible for inclusion, patients had to fulfil the DSM-IV (American Psychiatric Association, 1994) criteria for a current major depressive episode. We excluded patients with neurological disorders, those receiving medications with potential central nervous system side-effects and those with uncontrolled medical illness at the time of recruitment. Psychiatric exclusion criteria included any history of mania, hypomania or non-affective psychosis, and current substance dependence. Similarly, in order to reduce the risk that prior exposure to electroconvulsive therapy (ECT) might bias the results, we excluded patients who had received ECT within 6 months of recruitment.

The study was approved by the ethical committee of our hospital. All patients (or a close relative when there was doubt about the patient's understanding) gave informed written consent.

\section{Clinical assessment}

Depressive symptoms were rated on the Spanish version of the 17-item Hamilton Rating Scale for Depression (HRSD; Hamilton, 1960; Ramos-Brieva \& Cordero-Villafafila, 1988) and on the Newcastle Scale (Carney et al, 1965). All patients recruited had a baseline HRSD score of 21 or greater. A baseline Newcastle Scale score of 7 or higher was used to define endogenous depression. We also screened for reduced global cognitive function using the Spanish version of the Mini-Mental State Examination (MMSE; Folstein et al, 1975; Lobo et al, 1999).

Following Baldwin \& Tomenson (1995), we quantified vascular risk factors as follows: 0, absent; 1 , mild (asymptomatic), for example, controlled hypertension or clinical evidence of arteriosclerosis (e.g. carotid murmur); 2, moderate (symptomatic), for example, poorly controlled hypertension, symptomatic angina or history of myocardial infarction; and 3, severe (active disease), for example, peripheral vascular disease with amputation or transient ischaemic attack. Other vascular risk factors such as blood levels of cholesterol and glucose were also assessed.

\section{Study design}

To minimise bias from the administration of psychotropic drugs, patients underwent a minimum washout period of 10 days for antidepressant medication and a minimum of 2 days for benzodiazepine medication. Baseline clinical assessments, including brain single photon emission computed tomography (SPECT), were then conducted. After the SPECT scan, treatment was started. Fortnightly clinical evaluations (HRSD) were made during the study. 
Evaluators were masked to the functional neuroimaging measures. Patients were considered to be in remission when the 17-item HRSD score was below 8 on two consecutive visits.

\section{Treatment protocol}

The treatment used complied with standard therapeutic norms, and specifically with our hospital's treatment protocol for elderly patients with major depression. The antidepressant initially used was nortriptyline at a daily dosage of $12.5 \mathrm{mg}$, which was raised to $25 \mathrm{mg}$ and then $50 \mathrm{mg}$ on the fourth and eighth day of treatment, respectively. Nortriptyline plasma concentrations were determined after 1 week of stable dosing; the dose was adjusted to obtain a blood level between $80 \mathrm{ng} / \mathrm{ml}$ and $120 \mathrm{ng} / \mathrm{ml} \mathrm{(304-}$ $456 \mathrm{nmol} / \mathrm{l})$; maximum dose $100 \mathrm{mg} /$ day. All patients achieved correct plasma levels during the first month of treatment. In combination with antidepressant treatment, both haloperidol (up to $4 \mathrm{mg} /$ day for management of psychotic symptoms) and lorazepam (up to $4 \mathrm{mg}$ /day for management of anxiety/insomnia) were allowed.

Patients remained on a regimen of nortriptyline for 12 weeks. Patients whose HRSD score had not fallen by at least $50 \%$ by the end of the sixth week of nortriptyline treatment received adjunctive citalopram $(20 \mathrm{mg} / \mathrm{day})$ until the end of the 12-week follow-up.

\section{SPECT procedure}

At the end of the drug wash-out period, each participant underwent a ${ }^{99 \mathrm{~m}}$-technetium hexamethylpropylene amine oxime $\left({ }^{99 \mathrm{~m}} \mathrm{Tc}-\right.$ HMPAO) SPECT brain scan. The scan was performed using a rotating dual-head gamma camera (Helix, General Electric Medical Systems), fitted with a highresolution fanbeam collimator. Data acquisition started $20 \mathrm{~min}$ after intravenous injection of $740 \mathrm{MBq}{ }^{99 \mathrm{~m}} \mathrm{Tc}-\mathrm{HMPAO}$ (Ceretec; Nycomed, Amersham, UK). Sixty $30 \mathrm{~s}$ frames were collected in a $360^{\circ}$ circular orbit, step and shoot mode, using a $128 \times 128$ matrix.

Image data were processed on an Elscint SP1 computer (Apex SP-X, software version 3.12). Reconstruction was performed by filtered back-projection using a Metz filter (power factor 3). No attenuation correction was performed. The final pixel size was $3.9 \mathrm{~mm}$ and the full width at half-maximum in the transaxial plane was $10 \mathrm{~mm}$. Semi-quantitative regional cerebral blood flow analysis was performed using irregular regions of interest (ROIs) stored in the computer as a $T_{1}$-weighted magnetic resonance imaging (MRI) template. Brain SPECT images were normalised to the standard $T_{1}$-weighted MRI. The ROIs were placed in ten 7.8-mm-thick oblique slices, which were parallel to the fronto-cerebellar plane. For each hemisphere, anterior fronto-cerebellar ratios were obtained as $100 \times$ mean counts per pixel of anterior frontal ROIs divided by the mean counts per pixel of cerebellar ROIs. Using the same formula, the posterior fronto-cerebellar ratios were also obtained. The nuclear medicine physician was masked to the 12 -week course.

\section{Statistical procedures}

Comparisons between groups were assessed by using the Student's $t$-test (or the MannWhitney $U$-test for non-parametric data) for continuous variables and the chisquared test for discrete variables. The Kolmogorov-Smirnov test was applied to assess the normality of the continuous variables. The level of statistical significance was set at $P<0.05$.

Logistic regression methods were used to define a predictive model of non-remission. In the first stage, only the frontal perfusion measures were assessed. In the second stage, the baseline functional neuroimaging variables and the baseline clinical and demographic variables were assessed jointly to improve the model. Different models were obtained and explored by forward selection, backward selection and stepwise regression. The most significant variables were then fixed in the model and the others were introduced progressively to improve the adjustment. The model calibration was assessed by the HosmerLemeshow goodness-of-fit test. Finally, the reliability and precision of each model were analysed in order to choose the best final solution (Doménech, 1999).

To avoid any possible separation between groups confounding the results, the predictive models were assessed further by using a split-half approach. To try to avoid an unlucky splitting of the samples that would cause some variables to be absent in certain groups, the following approach was adopted. The whole sample was split randomly into two groups, $\mathrm{A}$ and $\mathrm{B}$. To increase the possibility that the data-sets to be analysed covered the complete variation, two more groups were generated, $\mathrm{C}$ and D. Group $\mathrm{C}$ was made from the first half of $A$ and $B$, and group $D$ from the last half of A and B. Every model was fitted to each of the data-sets. If the solution replicates over sets $\mathrm{A}$ and $\mathrm{B}$ or over sets $\mathrm{C}$ and $\mathrm{D}$, the possibility of empirical verification of correctness of the solution (i.e. that the model captures essential variation not only pertaining to the specific samples) is increased. By contrast, although the absence of stability of some components across subsamples might reflect its presence only in specific subsets, this finding increases the probability that the model will not be valid.

Finally, for further clarification of our findings in a clinical sense, the receiver operating characteristic (ROC) curve was measured for each continuous variable to define the cut-off point with the best sensitivity and specificity.

\section{RESULTS}

The initial sample comprised 52 patients, five of whom were eventually excluded: one had died, two did not attend scheduled follow-up visits, and two required ECT because of severe clinical deterioration. The final sample therefore comprised 47 patients.

The Kolmogorov-Smirnov test showed that three variables (MMSE score, Newcastle Scale score and the previous evolution in years) did not adjust to a normal distribution. It was possible to calculate a logarithmic transformation of the Newcastle score for adjustment to a normal distribution. It was not possible to transform the MMSE score or the years of previous evolution for adjustment to a normal distribution.

The mean age was 74.28 (s.d. $=7.68$, range 62-89) years. Approximately twothirds of patients were female $(68 \%)$. Patients' symptoms were moderate to severe, as shown by their HRSD scores (mean 31.04 , s.d. $=6.25$ ) and by the incidence rates of endogenous features $(32 \%)$, psychotic features $(19 \%)$ and admissions to hospital $(23 \%)$.

Of the 47 patients who completed the study, 34 were classed as 'remitters' and 13 as 'non-remitters'. Some clinical data for both subgroups are presented in Table 1. Significant differences between the subgroups were found in the variables duration of current episode, Newcastle score, left anterior frontal ratio (LAFR) and right 
Table I Baseline demographic, clinical and neuroimaging characteristics of study sample

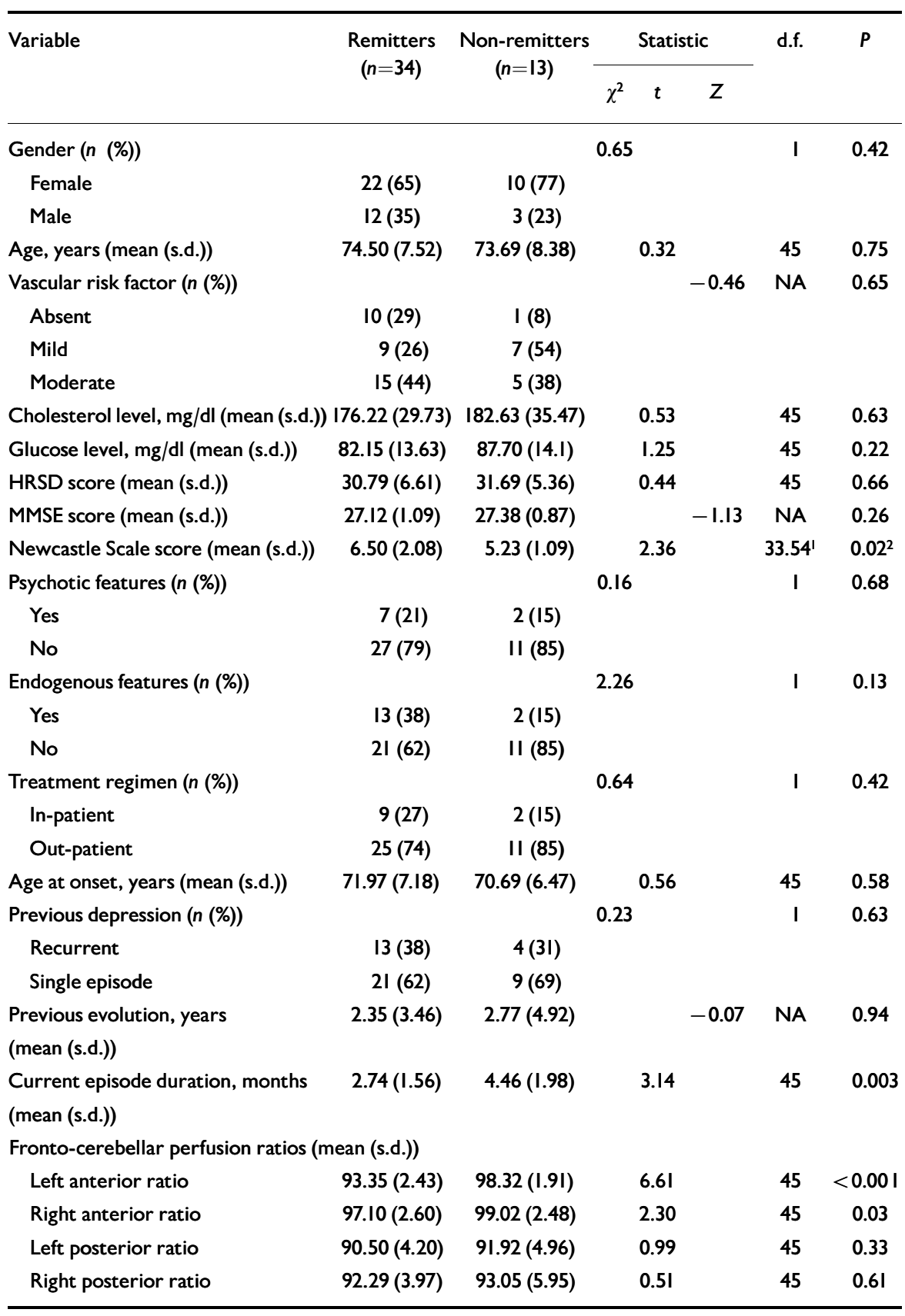

HRSD, Hamilton Rating Scale for Depression; MMSE, Mini-Mental State Examination; NA, not applicable.

I. Corrected for inequality of variances.

2. After transformation for adjustment to a normal distribution.

Table 2 Split-half analysis of the predictive model of non-remission with SPECT variables (LAFR)

\begin{tabular}{|c|c|c|c|c|c|c|c|c|c|c|c|}
\hline & \multicolumn{5}{|c|}{ Model statistics } & \multicolumn{6}{|c|}{ Variable statistics } \\
\hline & $\chi^{2}$ & d.f. & $P$ & $-2 \ln R$ & $\begin{array}{c}\text { Nagelkerke } \\
R^{2}\end{array}$ & $\begin{array}{c}\text { Wald } \\
\chi^{2}\end{array}$ & d.f. & $P$ & $\begin{array}{l}\text { Change } \\
-2 \ln R\end{array}$ & d.f. & $P$ \\
\hline Group A & 15.54 & I & $<0.001$ & 11.45 & 0.71 & 4.84 & I & 0.03 & 15.54 & I & $<0.001$ \\
\hline Group B & 15.75 & I & $<0.001$ & 12.52 & 0.70 & 5.51 & I & 0.02 & 15.75 & I & $<0.001$ \\
\hline Group C & 5.67 & I & 0.02 & 8.1 & 0.48 & 3.9 & I & 0.08 & 5.67 & I & 0.02 \\
\hline Group D & $\mathbf{I 7 . 7 8}$ & 1 & $<0.001$ & 14.06 & 0.72 & 7.06 & I & $<0.01$ & 17.78 & I & $<0.001$ \\
\hline
\end{tabular}

SPECT, single photon emission computed tomography; LAFR, left anterior frontal ratio. anterior frontal ratio. No significant difference was found in other clinical variables such as age of onset, age of index episode or presence of vascular risk factors, or in neuroimaging variables such as left and right posterior frontal ratios (Table 1).

\section{Predictive model of non-remission with frontal perfusion measures}

Of the four frontal areas assessed (left and right anterior frontal and left and right posterior frontal) the predictive model included only the LAFR (Wald $\chi^{2}=11.88$; d.f. $=1, P<0.01)$. The model explained $67.8 \%$ of the proportion of uncertainty of data $\left(-2 \operatorname{lnR}=25.65 ; \quad \chi^{2}=29.78 ; \quad\right.$ d.f. $=1$; $P<0.01$; Nagelkerke $R^{2}=0.678$ ). Its goodness of fit was acceptable $\left(\chi^{2}=2.66\right.$; d.f. $=7 ; P=0.92)$ and the predictions showed good sensitivity $(76.9 \%)$ and specificity $(91.2 \%)$. The global predictive power was $87.2 \%$. The inclusion of the LAFR indicated that the risk of non-remission at 3-month follow-up rose almost 2.5 times with every point that the LAFR increased $(\exp (B)=2.54 ; 95 \%$ CI $1.5-4.32)$. In the split-half approach, the only variable entering the four subsamples was the LAFR. The solution was found to be replicated over subsets (Table 2 ).

The ROC curve measurement for the LAFR showed that the best cut-off point was a ratio value of 95.5 (sensitivity $92.3 \%$; specificity $88.2 \%$ ); area under the curve $=0.95 ; 95 \%$ CI $0.89-1 ; P<0.01$ ).

\section{Global predictive model of non-remission}

To improve the predictive model, the second stage assessed both the baseline functional neuroimaging variables and the baseline clinical and demographic variables. The global predictive model included LAFR (Wald $\chi^{2}=6$; d.f. $=1 ; P<0.01$ ), current episode duration (Wald $\chi^{2}=4.57$; d.f. $=1$, $P=0.03$ ) and current age (Wald $\chi^{2}=3.31$; d.f. $=1 ; \quad P=0.07)$. Although statistically non-significant, current age increased the proportion of uncertainty explained by the model significantly $(75 \%$ to $83 \%)$ and in particular improved the Hosmer-Lemeshow goodness of fit $\left(\chi^{2}\right.$ significance from $P=0.58$ to $P=0.99$ ). Although lacking clinical significance, current age was finally included for methodological adjustment. This global model explained $83 \%$ of the proportion of uncertainty of data $(-2 \ln R=15.08$; $\chi^{2}=40.35 ;$ d.f. $=3 ; \quad P<0.01$; Nagelkerke $R^{2}=0.832$ ), its goodness of fit was 
appropriate $\left(\chi^{2}=0.96\right.$; d.f. $\left.=7 ; P=0.99\right)$ and its predictions showed good sensitivity $(92.3 \%)$ and specificity $(94.1 \%)$. The global predictive power was $93.6 \%$.

The most determinant variable was the LAFR. The risk of non-remission at 3month follow-up rose almost sixfold for each point that the LAFR increased $(\exp (\mathrm{B})=5.65 ; 95 \%$ CI $1.41-22.61)$. The second variable entering the final model was the current episode duration, indicating that the risk of non-remission increased almost fourfold for every extra month in the current episode duration $(\exp (\mathrm{B})=3.81$; $3.81 ; 95 \%$ CI 1.12-12.93). In the split-half approach, the only variable entering the four subsamples was again the LAFR. The solution including the clinical variables was not replicated over subsets (Table 3).

The best cut-off point for current episode duration according to the ROC curve measurement was 2.5 months (sensitivity $84.6 \%$; specificity $55.9 \%$; area under the curve $=0.76 ; 95 \%$ CI $0.61-0.9 ; P=0.007$ ).

\section{DISCUSSION}

The main findings of this study are that:

(a) The LAFR seems to be able to predict response to acute pharmacological treatment in late-onset major depression.

(b) Adding baseline clinical variables, duration of index episode and current age to the LAFR in the statistical analysis seems to increase the model's predictive power. However, these results are not confirmed by the split-half approach, and could be due to the sample characteristics.

\section{Variables predicting outcome}

Our results suggest that the risk of nonremission at 3-month follow-up increases almost 2.5 times with each extra point in the LAFR. The cut-off point of 95.5 predicts with an acceptable sensitivity and specificity the remission/non-remission of the depressive episode after 3 months of pharmacological treatment. Unlike structural neuroimaging studies of patients with late-onset major depression, which have described an association between frontal structural alterations and response to treatment (Simpson et al, 1998; Alexopoulos et al, 2002), to our knowledge this is the first functional neuroimaging study to report an association between cerebral perfusion and response to pharmacological treatment in this subgroup of patients with depression. The stability of the SPECT variable for predicting the therapeutic outcome across the split-half approach increases the possibility of correctness of the model.

Furthermore, in our study, the addition of the duration of the index episode and current age seemed to increase the model's predictive ability. However, taking into account that these results were not confirmed in the split-half approach, the absence of stability of some clinical components across subgroups increases the probability that the model will not be valid and challenges the conclusions obtained from its use. In either case, further studies applying the same models on an entirely different data-set are required to confirm our findings.

The possible association between the clinical variables, duration of index episode and current age, and course of the illness has been assessed in a number of studies, but the results are inconsistent. Like us, a number of authors have found a correlation between older age at the time of the index episode and poor response to treatment (Kennedy et al, 1991; Dew et al, 1997), although the results of other studies have not corroborated this claim (Burvill et al, 1991; Tuma, 1996; Halloran et al, 1999; Bosworth et al, 2002). Equally, our finding of a correlation between longer duration of current episode and poorer response to treatment is in agreement with some earlier studies (Murphy, 1983; Tuma, 1996; Halloran et al, 1999), but not with others (Burvill et al, 1991; Kennedy et al, 1991; Dew et al, 1997; Bosworth et al, 2002). This lack of consensus is probably caused by the wide range of methodological approaches, statistical analyses and demographic characteristics of the samples in these studies.

Regarding other clinical variables, it should be emphasised that the existence of significant differences in the Newcastle Scale scores between remitters and nonremitters (higher scores among remitters; Table 1) seems consistent with most of the previous studies suggesting higher rates of response to acute antidepressive treatment in patients with endogenous depression compared with patients with non-endogenous depression (Nelson \& Charney, 1980; Schatzberg, 1999). At this point, it is not possible to know whether increasing the size of our study sample would have incorporated the Newcastle clinical variable in the global predictive model.

Table 3 Split-half analysis of the global predictive model of non-remission

\begin{tabular}{|c|c|c|c|c|c|c|c|c|c|c|c|}
\hline & \multicolumn{5}{|c|}{ Model statistics } & \multicolumn{6}{|c|}{ Variable statistics } \\
\hline & $\chi^{2}$ & d.f. & $P$ & $-2 \ln R$ & Nagelkerke $R^{2}$ & Wald $\chi^{2}$ & d.f. & $P$ & Change $-2 \ln R$ & d.f. & $P$ \\
\hline Group A & 15.54 & 1 & $<0.001$ & 11.45 & 0.71 & & & & & & \\
\hline LAFR & & & & & & 4.84 & 1 & 0.03 & 15.54 & 1 & $<0.001$ \\
\hline Group B & 22.2 & 2 & $<0.001$ & 6.06 & 0.87 & & & & & & \\
\hline LAFR & & & & & & 1.44 & 1 & 0.23 & 17.91 & 1 & $<0.001$ \\
\hline Newcastle & & & & & & 1.12 & 1 & 0.29 & 6.46 & 1 & 0.01 \\
\hline Group C & 5.67 & 1 & 0.02 & 8.1 & 0.48 & & & & & & \\
\hline LAFR & & & & & & 3.9 & 1 & 0.08 & 5.67 & 1 & 0.02 \\
\hline Group D & 24.22 & 2 & $<0.001$ & 7.62 & 0.87 & & & & & & \\
\hline LAFR & & & & & & 3.17 & 1 & 0.07 & 11.66 & 1 & 0.001 \\
\hline Current episode duration & & & & & & 1.49 & I & 0.22 & 6.44 & I & 0.01 \\
\hline
\end{tabular}

LAFR, left anterior frontal ratio. 


\section{Significance of frontal perfusion abnormalities in late-onset major depression}

It is not easy to explain why the lower the LAFR (i.e. the higher the left anterior frontal hypoperfusion) the better the rates of clinical remission. It could be that the LAFR reflects only the speed of response to pharmacological treatment and not remission/non-remission. A longer followup of the patients, especially bearing in mind the evidence that geriatric patients might take longer to respond to antidepressant treatment (Mulsant \& Pollock, 1998; Reynolds et al, 1996), would provide more information on this issue. It is difficult to establish the histological and clinical significance of the cut-off point described; in any case, we believe that its significance lies in its usefulness as a prognostic factor and as a variable in the area of research.

We should stress that the direction of the prediction is at the very least surprising, especially as the few descriptive studies using functional neuroimaging to investigate acute major depression in the elderly have obtained inconsistent results (Ebmeier et al, 1998; Halloran et al, 1999; Navarro et al, 2002). These inconsistencies suggest that the alterations in frontal perfusion in this disorder are not specific.

The frontal functioning neuroimaging results of our study do not support our initial hypothesis and therefore do not corroborate the proposal of Alexopoulos et al (1997) of a degenerative ischaemic process as the cause of late-onset major depression (vascular depression). Our hypothesis was that the greater the frontal hypoperfusion (supposedly a manifestation of cerebral ischaemia), the lower would be the level of clinical reversibility (that is, a lower rate of remitters). This study found the reverse to be the case.

To explain this unexpected result, we could state that most studies of vascular depression are associative (O'Brien et al, 1996; Greenwald et al, 1998) rather than causative, and it is possible that the frequently observed association between late-onset major depression and vascular disease could in fact be the result of unknown confounding factors. A vascular aetiology has been proposed for late-onset depression on the strength of the presence of hyperintensities, although the physiopathological bases of these lesions have not been determined definitively in patients with major depression (Thomas et al,

\section{CLINICAL IMPLICATIONS}

- The presence of a high level of left anterior frontal perfusion suggests poor acute antidepressant response in late-onset severe major depression.

- The frontal lobes, especially the left anterior frontal area, seem to be linked to the physiopathology of late-onset severe major depression.

The relationship between late-onset major depression and vascular depression is an important issue of which little is known at present.

\section{LIMITATIONS}

- As this is a study of predictive factors, a larger sample size would have been desirable.

Only patients with late-onset and severe depression were included in our sample, meaning that the results cannot be extrapolated to the general population or even to the general elderly population.

- Treatment was administered in a naturalistic manner.

VÍCTOR NAVARRO, MD, PhD, CRISTÓBAL GASTÓ, MD, PhD, Clinical Institute of Psychiatry and Psychology, Hospital Clinic, Barcelona, Spain; FRANCISCO LOMEÑA, MD, PhD, Department of Nuclear Medicine, Hospital Clinic, Barcelona, Spain; XAVIER TORRES, PsyD, Clinical Institute of Psychiatry and Psychology, Hospital Clinic, Barcelona; JOSÉ J. MATEOS, MD, Department of Nuclear Medicine, Hospital Clinic; MARÍA PORTELLA PsyD, GUILLEM MASANA, MD, PhD, TEODORO MARCOS, PsyD, PhD, Clinical Institute of Psychiatry and Psychology, Hospital Clinic, Barcelona, Spain

Correspondence: Dr Víctor Navarro, Hospital Clínic, Departamento de Psiquiatría, Villarroel 170, 08036 Barcelona, Spain. Tel: 343 2275477; fax: 343 2275477; e-mail: 30739vno@comb.es

(First received 14 April 2003, final revision 28 July 2003, accepted 5 November 2003)

2002). We should add that this is not the first study to obtain results that challenge the degenerative vascular hypothesis for late-onset major depression (Dupont et al, 1995; Ebmeier et al, 1997; Greenwald et al, 1998; Navarro et al, 2002). Nonetheless, we should stress that the inferences made in this section, i.e. that our results do not support the vascular depression hypothesis, are based on the possible but unproven association between brain SPECT alteration and ischaemia. This association is debatable, given the absence of conclusive studies that support its existence in major depression.

Our results suggest that the pattern of left anterior frontal perfusion reflects a primary alteration of neuronal activity, probably reversible, and that there might not necessarily be a primary vascular substrate. Equally, although future studies should test the predictive models obtained here, this report provides strong evidence in favour of the existence of specific neurofunctional predictors of the course in lateonset severe major depression.

\section{REFERENCES}

Alexopoulos, G. S., Kiosses, D. N., Choi, S. J., et al (2002) Frontal white matter microstructure and treatment response of late-life depression: a preliminary study. American Journal of Psychiatry, 159, 1929-1932.

Alexopoulos, G. S., Meyers, B. S., Young, R. C., et al (1997) "Vascular depression" hypothesis. Archives of General Psychiatry, 54, 915-922.

Alexopoulos, G. S., Meyers, B. S., Young, R. C., et al (2000) Executive dysfunction and long-term outcomes of geriatric depression. Archives of General Psychiatry, 57, 285-290.

American Psychiatric Association (1994) Diagnostic and Statistical Manual of Mental Disorders (4th edn) (DSM-IV). Washington, DC: APA.

Baldwin, R. C. \& Tomenson, B. (1995) Depression in later life. A comparison of symptoms and risk factors in early and late onset cases. British Journal of Psychiatry, 167, 649-652. 
Bosworth, H. B., Hays, J. C., George, L. K., et al (2002) Psychosocial and clinical predictors of unipolar depression outcome in older adults. International Journa of Geriatric Psychiatry, I7, 238-246.

Burvill, P. W., Hall, W. D., Stampfer, H. G., et al (199I) The prognosis of depression in old age. British Journal of Psychiatry, 158, 64-71.

Carney, M. P. W., Roth, M. \& Garside, R. F. (1965) The diagnosis of depressive syndromes and the prediction of ECTresponse. British Journal of Psychiatry, III, 659-674.

Dew, M. A., Reynolds, C. F., Houck, P. R., et al (1997) Temporal profiles of the course of depression during treatment. Predictors of pathways toward recovery in the elderly. Archives of General Psychiatry, 54, 1016-1024.

Doménech, J. M. (1999) Construcción de un modelo de regresión logística. In: Análisis Multivariante en Ciencias de la Salud. Barcelona: Editorial Signo.

Dupont, R. M., Butters, N., Schafer, K., et al (1995) Diagnostic specificity of focal white matter abnormalities in bipolar and unipolar mood disorder. Biological Psychiatry, 38, 482-486.

Ebmeier, K. P., Prentice, N., Ryman, A., et al (1997) Temporal abnormalities in dementia and depression: a study using high resolution single photon emission tomography and magnetic resonance imaging. Journal of Neurology, Neurosurgery and Psychiatry, 63, 597-604.

Ebmeier, K. P., Glabus, M. F., Prentice, N., et al (1998) A voxel-based analysis of cerebral perfusion in dementia and depression of old age. Neuroimage, 7, 199-208.

Folstein, M. F., Folstein, S. E. \& McHugh, P. R. (1975) Mini-Mental State: a practical method for grading the cognitive state of patients for the clinician. Journal of Psychiatric Research, 12, 189-198.
Greenwald, B. S., Kramer-Ginsberg, E., Krishnan, K. R., et al (1998) Neuroanatomic localization of magnetic resonance imaging signal hyperintensities in geriatric depression. Stroke, 29, 613-617.

Halloran, E., Prentice, N., Murray, C. L., et al (1999) Follow-up study of depression in the elderly. Clinical and SPECT data. British journal of Psychiatry, 175, 252-258.

Hamilton, M. (1960) A rating scale for depression. Journal of Neurology, Neurosurgery and Psychiatry, 23 $56-62$

Kennedy, G. J., Kelman, H. R. \& Thomas, C. (1991) Persistence and remission of depressive symptoms in late-life. American Journal of Psychiatry, 148, 174-178.

Kumar, A., Thomas, A., Lavretsky, H., et al (2002) Frontal white matter biochemical abnormalities in latelife major depression detected with proton magnetic resonance spectroscopy. American Journal of Psychiatry, 159, 630-636.

Lobo, A., Saz, P., Marcos, G., et al (1999) Revalidation and standardization of the cognition mini-exam (first Spanish version of the Mini-Mental Status Examination) in the general geriatric population. Medicina Clinica, II2 767-774.

Mulsant, B. H. \& Pollock, B. G. (1998) Treatmentresistant depression in late life. Journal of Geriatric Psychiatry and Neurology, II, 186-193.

Murphy, E. (1983) The prognosis of depression in old age. British Journal of Psychiatry, 142, III-119.

Navarro, V., Gastó, C., Lomeña, F., et al (2002)

Normalization of frontal cerebral perfusion in remitted elderly major depression: a 12-month follow-up SPECT study. Neuroimage, 16, 781-787.
Nelson, J. C. \& Charney, D. S. (1980) Primary affective disorder criteria and the endogenous-reactive distinction. Archives of General Psychiatry, 37, 787-793.

O'Brien, J., Desmond, P., Ames, D., et al (1996) A magnetic resonance imaging study of white matter lesions in depression and Alzheimer's disease. British Journal of Psychiatry, 168, 477-485.

Reynolds, C. F., Frank, E., Kupfer, D. J., et al (1996) Treatment outcome in recurrent major depression: a post hoc comparison of elderly ('young old') and midlife patients. American Journal of Psychiatry, 153, 1288-1292.

Ramos-Brieva, J. A. \& Cordero-Villafafila, A. (1988) A new validation of the Hamilton Rating Scale for Depression. Journal of Psychiatric Research, 22, 21-28.

Schatzberg, A. F. (1999) Antidepressant effectiveness in severe depression and melancholia. Journal of Clinical Psychiatry, 60, 14-21.

Simpson, S., Baldwin, R. C., Jackson, A., et al (1998) Is subcortical disease associated with a poor response to antidepressants? Neurological, neuropsychological and neuroradiological findings in late-life depression. Psychological Medicine, 28, 1015-1026.

Thomas, A. J., O'Brien, J.T., David, S., et al (2002) Ischemic basis for deep white matter hyperintensities in major depression: a neuropathological study. Archives of General Psychiatry, 59, 785-792.

Tuma, T. A. (1996) Effect of age on the outcome of hospital treated depression. British Journal of Psychiatry, 168, 76-81. 